Erschienen in: Blühdorn, Hardarik/Breindl, Eva/Waßner, Ulrich W. (Hrsg.):

Text - Verstehen. Grammatik und darüber hinaus. - Berlin, New York: de

Gruyter, 2006. S. 315-331. (Institut für Deutsche Sprache. Jahrbuch 2005),

https://doi.org/10.1515/9783110199963.2.315

\author{
EVA-MARIA JAKOBS
}

\title{
Texte im Berufsalltag: Schreiben, um verstanden zu werden?
}

\begin{abstract}
Der Beitrag fokussiert Textverständlichkeit aus der Sicht des Schreibens am Arbeitsplatz. Er vertritt die These, dass es im beruflichen Alltag häufig weniger um die Verständlichkeit des Produkts geht als um andere Ziele, wie die Absicherung des Verfassers. Die Kategorie Verständlichkeit wird status-, situations- und kontextabhängig unterschiedlich gehandhabt. Der Produktionsprozess wie auch das Produkt werden wesentlich durch innere und äußere Bedingungen des Arbeitskontextes geprägt. An Beispielen aus verschiedenen Berufswelten wird gezeigt, wie sich Status, Zeitdruck und psychischer Stress, subjektive Theorien über Textfunktionen und Adressaten, Arbeitsaufgabe und -organisation sowie Tendenzen der Arbeitswelt wie die Industrialisierung des Schreibens auf Schreibprozesse und ihre Produkte auswirken. Sie erzeugen berufs- und domänenspezifische wie auch übergreifende Phänomene mit z.T. weitreichenden Folgen für die Wirtschaft. Probleme entstehen vor allem bei schwierigen Themen, emotionaler Betroffenheit, konfligierenden Zielen und Mehrfachadressierung. Die Diskussion stützt sich auf kognitiv-textlinguistisch fundierte Modelle des Textproduzierens sowie 180 Interviews mit Vertretern verschiedener beruflicher Domänen.
\end{abstract}

\section{Vorbemerkung}

Streben nach Textverständlichkeit setzt voraus, dass die kommunikative Aufgabe Verständlichkeit verlangt und/oder dass der Textproduzent diese als ziel- und handlungsleitendes Konstrukt begreift. Betrachtet man Textverständlichkeit aus der Perspektive beruflichen Schreibens, so zeigt sich, dass dies häufig nicht oder nur bedingt der Fall ist.

Die Produktionsperspektive ist für das Thema Textverständlichkeit in verschiedener Hinsicht ergiebig. Sie ermöglicht Einblicke in die Konzepte und Ziele, die die Entstehung von Texten steuern, z. B. Stilvorstellungen. Sie lenkt den Blick auf die Hintergründe dieser Konzepte, z. B. Ausbildung, Sozialisation und Status. Und: sie schärft den Blick für das komplexe Bedingungsgefüge, in dem Texte entstehen, z.B. für Kontextbedingungen, die das Kriterium der Verständlichkeit mitunter in den Hintergrund rücken lassen.

Im vorliegenden Beitrag wird an Beispielen aus verschiedenen beruflichen Kontexten diskutiert, wie sich Arbeitsaufgabe und -organisation, interpersonale Beziehungen und subjektive Theorien über Textfunktionen und Adressaten auf die Verfertigung von Texten und deren Beschaffenheit auswirken. Zum Teil handelt es sich um berufs- bzw. domänenspezifische Phänomene, zum Teil um domänenübergreifende Tendenzen. 


\section{Theoretischer Hintergrund und Datenbasis}

\subsection{Modellierung des Schreibprozesses}

Die Diskussion stützt sich auf Konzepte zum Schreiben am Arbeitsplatz, die in Jakobs $(1999,2005$ a) ausführlich beschrieben sind. Das Basismodell (Jakobs 1999) beschreibt das Verfassen von Texten als iterativen Handlungskomplex, in dem produktive, reproduktive und rezeptive Prozesse interagieren (vgl. Abb.1). Sie dienen dem Abruf von Informationen aus dem Gedächtnis oder aus externen Quellen, der Bewertung, Strukturierung und Linearisierung der bereitgestellten Informationen, der Entwicklung mentaler Prä-Formulierungen, ihrer Externalisierung, Kontrolle und Korrektur. Gesteuert wird der Prozess durch intern repräsentierte Schemata und Ziele des Schreibers. Dazu gehören z. B. auch die Orientierung auf den Adressaten und die Absicht, sich verständlich auszudrücken.

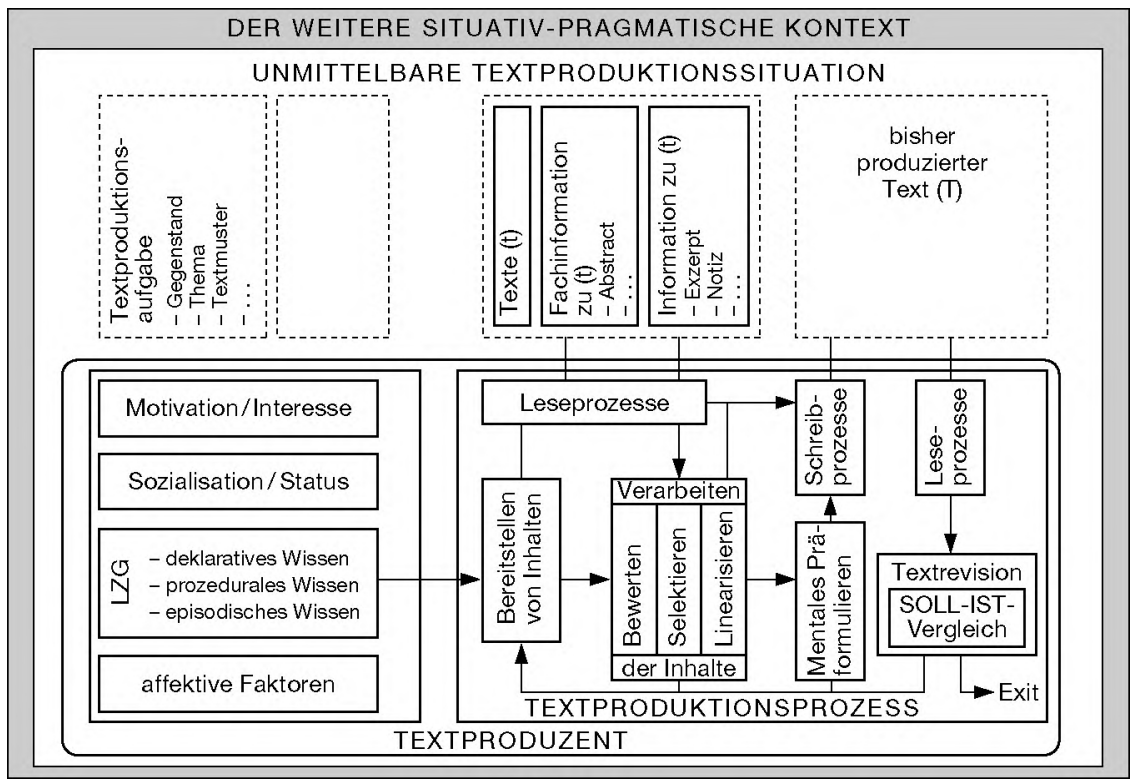

Abb. 1: Textproduktionsprozess (nach Jakobs 1999)

Die Ausführung von Textproduktionsprozessen (Art, Inhalt und Abfolge der beteiligten Teilprozesse) und - über diese - die Qualität des Produkts hängt von verschiedenen Faktoren ab. Dazu gehören u. a. affektive Faktoren, wie Interesse, Motivation, Betroffenheit, sowie die Schreibaufgabe. Mit Blick auf die Vielfalt der in der Praxis schriftlich realisierten Arbeitsanteile werden im

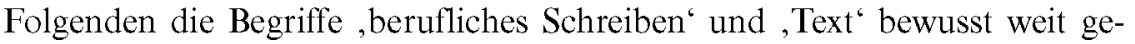
fasst. Sie subsumieren verschiedenartige Phänomene wie die weit verbreitete Praxis des Notizenmachens, das Ausfüllen von Formularen, das Schreiben 
von E-Mails, das Kommentieren von Schriftstücken oder das Verfassen von Dokumenten.

Schreiben im Beruf ist eine Form (und Ausdruck) des fachlichen Denkens und Handelns und als solche situiert: Die Entstehung von Texten vollzieht sich in einem komplexen Bedingungsgefüge von Faktoren, die in Abbildung 2 stark verallgemeinernd in einem Schalenmodell zusammengefasst sind. Jede Schale umfasst Komplexe sich beeinflussender Faktoren.

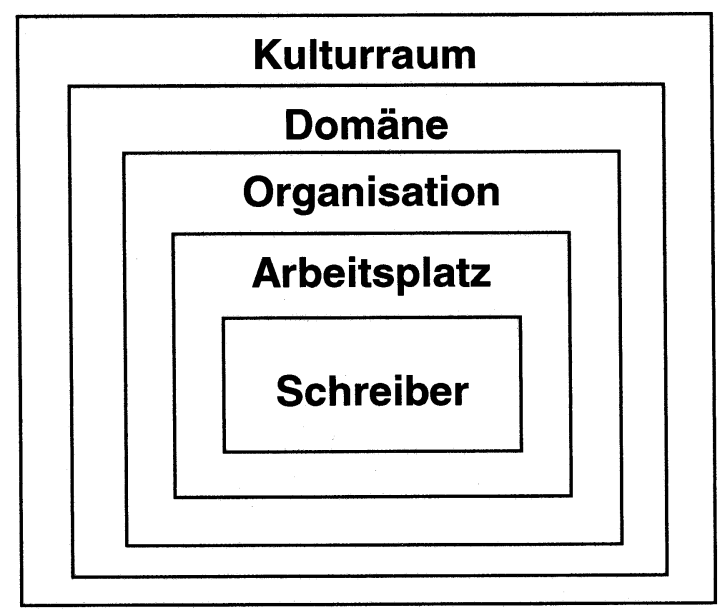

Abb. 2: Zusammenwirken interner und externer Faktoren (Jakobs 2005a)

Im Zentrum steht der Textproduzent in seiner Einbettung in Handlungskontexte. Der Handlungskontext umfasst Arbeitssituation und Arbeitsplatz, den übergeordneten organisationalen Rahmen, domänenspezifische Bedingungen sowie den Kulturraum, in dem die Beteiligten agieren.

$\mathrm{Zu}$ den personalen Größen gehören u. a. die Rolle des Berufsausübenden im Textproduktionsprozess (als Ideenspender, Verfasser, Koautor, Feedbackgebender etc.), seine Arbeitsaufgaben, die Position im Arbeitskontext (daran gebunden der Status), seine Expertise (Sach-, Schreib-, Medienkompetenz, Strategierepertoire), seine soziale, kulturelle und fachliche Sozialisation, Alter und Geschlecht, Einstellungen, Normen, Ziele und Motive (z. B. der Wunsch nach beruflichem Aufstieg), Medienpräferenz und -kompetenz sowie die mentale Repräsentation der Schreibaufgabe.

Die an den Arbeitsplatz und die Arbeitssituation gebundenen Größen betreffen die Textproduktionsaufgabe in ihrer Einbettung in übergeordnete textuelle und berufliche Aufgaben, Struktur und Organisation von Arbeitsabläufen, die Interaktion mit anderen (Kollege, Vorgesetzter), Teammerkmale (Art, Größe, Struktur, Normen und Kultur), die zur Verfügung stehenden Medien, die Raumbedingungen, das Zeit- und Kostenbudget, Vorgaben (Regeln, Mustertexte), andere Dokumente (Textentwürfe), relevante Textsorten u.a. 
Von Bedeutung ist die Einbettung in übergeordnete organisationale $\mathrm{Zu}-$ sammenhänge (z. B. eines Krankenhauses, eines Unternehmens, einer Organisation). Auf den Inhalt, den Ablauf und das Ergebnis des Textproduktionsprozesses wirken sich mehr oder weniger vermittelt Merkmale der Organisation (Art, Größe, Ziele, Struktur, Kultur, Produkt) aus, die globale und/oder lokale Ausrichtung der Organisation, Führungsstile, vertikale und horizontale Interaktionsbeziehungen (Kooperation, Konkurrenz), Vorgaben und Regeln, Fragen der Qualitätssicherung, das organisationale Medienmanagement, Dokumente (Mission Statement, Vorgaben, Checklisten, ISONormen etc.), Kommunikationsziele und -anlässe, Kommunikationskultur und -struktur, Kommunikationswege und -mittel sowie Kommunikationsregeln der Organisation.

Auch die Domäne selbst, d.h. die Branche oder das Berufsfeld, für die oder in der Texte entstehen, spielt eine Rolle. Hier werden Vereinbarungen über die Art und Weise beruflicher Interaktionen festgelegt. Die Domäne enthält kulturelle, soziale und ökonomische Normen bzw. Werte für die Interaktion mit Konkurrenten, Partnern (Zulieferer etc.) und Kunden.

Die Domänen sind Teil eines sozial, kulturell und zeitlich geprägten Raumes mit spezifischen Normen, Anspruchshaltungen und Erwartungen, gesetzlichen Vorgaben, Wertesystemen u. a.m. (vgl. dazu u. a. Fix 2005). Der Adressat kann in jeder der genannten Kontextebenen angesiedelt sein, im Falle der Dokumentation von Gebrauchsgütern z. B. erst in der äußersten Schale.

\subsection{Empirische Daten}

Die Diskussion stützt sich auf Daten des Forschungsschwerpunktes „Schreiben im Beruf " ${ }^{1}$. Ziel des Forschungsschwerpunktes sind Aussagen zu domänenspezifischen Textproduktionsprozessen und -produkten und deren Einbettung in situative Kontexte. Studien zum Gegenstand ermöglichen objektspezifische Modellierungen wie auch Aussagen zu domänenspezifischen Anforderungen und Problemen beruflichen Schreibens. Sie sind eine wesentliche Voraussetzung für die Definition von Berufsanforderungen, für die Entwicklung zielgruppengerechter Ausbildungs- und Trainingskonzepte sowie für die Gestaltung aufgabenbezogener elektronischer Arbeitsumgebungen. $\mathrm{Zu}$ allen drei Bereichen kann die Angewandte Linguistik einen wesentlichen Beitrag leisten.

Teil der Forschung ist der Aufbau einer Datenbasis zum Schreiben im Beruf. Die Daten werden mit verschiedenen Methoden gewonnen (Interview, Fragebogen, teilnehmende Beobachtung, Kontext- und Textanalyse, Auswertung der Fach- und Ratgeberliteratur u. a.). Der vorliegende Beitrag stützt sich

1 Vgl. www.tl.rwth-aachen.de. 
auf 180 halbstandardisierte Interviews mit Vertretern verschiedener Berufe. Die Interviews wurden zwischen Mai 2004 und Januar 2005 durchgeführt. ${ }^{2}$

\section{Die Ergebnisse: Schreiben am Arbeitsplatz}

\subsection{Umfang, Wichtigkeit, Anspruch und Fähigkeiten}

Unsere Daten zeigen, dass die Zielkategorie Textverständlichkeit berufs- und kontextbezogen unterschiedlich gehandhabt wird. Das dazugehörige Konzept variiert je nach Arbeitsinhalt, -kontext und -organisation sowie der Perspektive auf den Adressaten. Statushohe Berufsausübende legen deutlich mehr Wert auf Verständlichkeit als statusniedrige.

Domänenübergreifend zeichnet sich ab, dass das Schreiben von Texten zum Berufsalltag vieler, wenn nicht der meisten Berufe und Arbeitskontexte gehört. Wir baten die Befragten u. a. anzugeben, wie hoch sie den zeitliche Anteil und die Wichtigkeit von Schreibaufgaben bezogen auf ihren Arbeitsalltag einschätzen. Die Antworten deuten darauf, dass insgesamt erstaunlich viel geschrieben wird; in vielen Berufen und Arbeitsumgebungen wird Schreiben als wichtiger Bestandteil der Arbeit gewertet (vgl. Abb. 3; die Angaben beziehen sich auf kleine Stichproben, pro Berufsgruppe ca. 6 Probanden).

\begin{tabular}{|c|c|c|c|c|c|}
\hline Beruf & 1 & 2 & Beruf & 1 & 2 \\
\hline Bankkaufleute & +++ & $\uparrow$ & Ingenieure & ++ & $\uparrow$ \\
\hline Beamte (mittlerer Dienst) & +++ & $\uparrow$ & Psychologen (Therapeuten) & ++ & $\uparrow$ \\
\hline Journalisten & +++ & 4 & Feuerwehrleute & + & 4 \\
\hline Krankenschwestern & +++ & $\uparrow$ & Rettungssanitäter & + & $\uparrow$ \\
\hline Kriminalbeamte & +++ & $\uparrow$ & Ergotherapeuten & ++ & 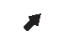 \\
\hline Mediziner & +++ & $\uparrow$ & Lehrer & ++ & 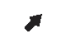 \\
\hline Notare & +++ & $\uparrow$ & Pfarrer & ++ & 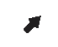 \\
\hline Öffentlichkeitsarbeiter & +++ & $\uparrow$ & Polizisten & ++ & 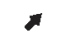 \\
\hline Rüstungsoffiziere & +++ & $\uparrow$ & Kindergärtner & + & 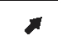 \\
\hline Steuerfachbearbeiter & +++ & $\uparrow$ & Krankengymnasten & + & $\pi$ \\
\hline Technische Redakteure & +++ & $\uparrow$ & Architekten & + & $x$ \\
\hline Wissenschaftler & +++ & $\uparrow$ & \multirow{2}{*}{$\begin{array}{l}\text { Postmitarbeiter (Zustellung, } \\
\text { Schalterdienst) }\end{array}$} & \multirow[t]{2}{*}{+} & \multirow[t]{2}{*}{$\mathbf{x}$} \\
\hline Betriebs- und Sozialpädagogen & ++ & 4 & & & \\
\hline
\end{tabular}

$1=$ Umfang: $+++:$ : $61-100 \%,++: 31-60 \%$, $+: 10-30 \%$

$2=$ Bedeutung: $\uparrow$ sehr wichtig. wichtig. eher weniger wichtig

Abb. 3: Berufsbezogener Umfang und Bedeutung des Schreibens

2 Die Interviews wurden vornehmlich von Studierenden durchgeführt, denen ich hier für ihr Engagement danken möchte. 
Die breite Etablierung elektronischer Kommunikationsmittel scheint berufsbezogen (kombiniert mit zunehmender Mobilität) dazu beizutragen, dass der Anteil schriftlich zu erledigender Arbeitsanteile tendenziell eher zu- als abnimmt (Dokumentationszwang, Email statt Telefonat, vgl. Jakobs (2005b, S. 17). In vielen Berufen wird der schriftlich zu leistende Anteil andererseits nach wie vor primär handschriftlich erledigt, z. B. im Rettungsdienst oder in der medizinischen Versorgung. Die Verständlichkeit leidet hier z.T. unter der Minimalforderung der Lesbarkeit. Im Falle medizinischer Anweisungen entsteht dadurch mitunter ein Sicherheitsrisiko.

Schriftsprachliche Ausdrucksfähigkeit ist häufig eine wesentliche Voraussetzung für Berufs- und Karrierechancen:

„Beruflicher Erfolg und berufliches Fortkommen erfordern die Fähigkeit, mit Texten umzugehen, wer es kann, kommt mit, wer darin versagt, bleibt sitzen oder geht unter, nicht nur individuell, sondern auch kollektiv, zusammen mit ganzen Wirtschaftszweigen, die im internationalen Wettbewerb abfallen." (Aebli 1983, S. 114)

Die Feststellung von Aebli wird in vielen Interviews bestätigt - mit der Verantwortung im Job nimmt der Anteil schriftlich zu bewältigender Aufgaben deutlich zu. In Leitungspositionen verdoppelt er sich in der Regel.

Die wenigsten Berufsausübenden (z. B. Juristen) werden auf die schriftlichen Anteile des Berufs vorbereitet. Dies gilt für akademische wie nicht-akademische Berufe. Dazu ein Zitat eines Mediziners, der in einem humangenetischen Institut arbeitet. Zu seinen Aufgaben gehört u.a. das Verfassen von Gutachten:

\begin{abstract}
„Das ist ja das völlig Diskrepante. Das Schreiben ist [...] ganz entscheidend in dem Beruf und im Studium hat man's eigentlich nie gelernt, weil im Prüfungssystem dieses schwachsinnige Multiple-Choice-System besteht. Man kreuzt halt an. [...] Kein Mensch lernt, selbstständig zu schreiben. Das rächt sich spätestens bei der [...] Doktorarbeit, [...] weil man keinen vernünftigen deutschen Satz formulieren kann. Das müsste verändert werden. [...] Es wird auch aus forensischen Gründen immer wichtiger, weil wenn es nämlich nicht gescheit dokumentiert ist, dann ist es auch nicht gescheit gemacht worden. Und kein Richter der Welt wird einem dann die Ausrede zulassen, ich konnte nichts ankreuzen, das konnte ich nicht dokumentieren." (Arzt - Institut für Humangenetik)
\end{abstract}

In einem Projekt zum Schreiben in den Ingenieurwissenschaften haben wir jeweils zwanzig Hochschullehrer und Assistenten der Fakultäten Maschinenbau und Elektrotechnik zu den schriftsprachlichen Ausdrucksfähigkeiten ihrer Studenten, zu Qualitätsanforderungen an Texte sowie zum Ausbildungsbedarf befragt. Die am häufigsten genannten Mängel betreffen Fähigkeiten, die zentral für das Verfassen verständlicher Texte sind: die Fähigkeit zu strukturieren sowie etwas auf den Punkt zu bringen, d.h. sich kurz und präzise auszudrücken. In diesem Zusammenhang werden u.a. Wortwahl, Grammatik und Zeichensetzung moniert.

Die genannten Mängel zeichnen sich in verschiedenen beruflichen Kontexten $a b$ und betreffen z.T. elementare Fähigkeiten wie die Beherrschung der 
Rechtschreibung. Mit der neuen Rechtschreibung haben übrigens die meisten der von uns befragten Berufsausübenden Probleme.

Ein spezifisches Ausbildungsproblem ergibt sich bei Berufswechseln. Häufig kommen die Berufsausübenden aus anderen, artfremden Berufen und müssen umlernen. Dazu ein Feuerwehrmann, der als Ausbilder die Hälfte seiner Zeit in Schreibaufgaben investiert:

„Von meinem Beruf her bin ich Schlosser, oder war mal Schlosser [...] Ich stell mich gerade um zu den Verwaltungssachen. Die Schreiberei ist mittlerweile mein Beruf. [...] Als Schlosser lernt man so etwas nicht." (Feuerwehrmann, Ausbilder)

\subsection{Copy and Paste - Übernehmen und Fortschreiben des Altbewährten}

Die meisten Berufsausübenden lernen das berufliche Schreiben erst in der Praxis. Die am häufigsten genannten Aneignungsstrategien sind „Learning by doing“ und „Copy and Paste“. Die Anfänger fragen erfahrene Kollegen, wie Texte aussehen sollen und/oder orientieren sich an Textbeispielen der beruflichen Umgebung (reproduzierendes, musterorientiertes Formulieren). Einige nutzen ergänzend die Ratgeberliteratur. Die genannten Strategien sind aus der Sicht der Qualität des Textprodukts mitunter kontraproduktiv.

Die Strategie des Copy and Paste setzt die existente Praxis fort und tradiert Stilvorstellungen der Amtsvorgänger, die diese in den siebziger, achtziger oder neunziger Jahren des 20. Jahrhunderts erworben haben. Die Strategie dürfte zu dem Phänomen beitragen, dass sich trotz Reformbemühungen häufig die Textpraxis des viel geschmähten, da als eher un- oder schwerverständlich bekannten Beamtendeutsch ausdauernd hält.

Die Orientierung an Sprachratgebern ist häufig auch nicht hilfreich - sie werden eher selten von echten Sprachexperten verfasst und zeichnen sich häufig durch verkürzende, generalisierende und trivialisierende Empfehlungen aus, wie z. B. allgemeines Passiv-Verbot oder generelle Aufwertung kurzer Wörter. Letzteres verdanken wir nicht zuletzt der Prominenz und Durchsetzungskraft des Hamburger Verständlichkeitskonzeptes von Langer/Schulz von Thun/Tausch (1975). Kurze Wörter werden zwar häufig schneller aufgenommen und verarbeitet, in vielen Fällen geht es beim Textverstehen jedoch weniger um die Schnelligkeit der Verarbeitung eines Ausdrucks als vielmehr um dessen Eignung, den Leser beim Aufbau eines mentalen Modells zu unterstützen.

Auch das Passiv-Verbot greift zu kurz. Wie Textberatung und -training zeigen, ist den wenigsten Sprachteilhabern bewusst, welches Leistungsspektrum das Passiv im Deutschen besitzt und dass es - textsortenabhängig - das bessere Mittel sein kann, z. B. wenn es in Geschäftsberichten darum geht, schlechte Nachrichten neutral zu verpacken (vgl. Keller 2004). Die Textsortenspezifik fehlt in den meisten Ansätzen zur Textverständlichkeit, insbesondere in Modellen von Psychologen. 
Interessant sind auch die subjektiven Stilvorstellungen, die sich im Verlauf des Berufslebens verändern können. Domänenspezifisch werten Anfänger mitunter Merkmale der Schwerverständlichkeit als fachlich guten Stil. Dazu ein Zitat von einem Hochschullehrer:

\begin{abstract}
„Die ersten Versuche des Schreibens, die waren: viel verdrehter, viel verschachtelter, viel, ich würd heute sagen, unklarer, und das hat lange gedauert, bis ich soweit war zu sagen, ne, das muss alles nicht sein, das kann und sollte einfacher gehen, und das war auch ein Akt der Befreiung. Ich hab nicht mehr den Anspruch, dass ich möglichst gedrechselt schreiben muss, sondern das Gegenteil. Das war noch zu den Zeiten, als ich promoviert hab, anders, da hab ich gedacht, dass die Wissenschaft sich dadurch auszeichnet, dass man möglichst gedrechselte Sätze schreibt." (Politologe, Hochschullehrer)
\end{abstract}

Die Kategorie Textverständlichkeit wird berufs-, kontext- und statusabhängig unterschiedlich interpretiert und gehandhabt. Statushohe Personen legen deutlich mehr Wert auf Verständlichkeit (vgl. auch Scheller 2005). Sie assoziieren mit Verständlichkeit vor allem Merkmale wie Kürze, Prägnanz und Klarheit des Ausdrucks.

"Was salopper schreiben kann man schon das eine oder andere Mal, wenn man Leute
nicht kennt; aber da wir den Anspruch insbesondere unserer jeweiligen Vorstände ken-
nen, unser Chef ist der Vorstandsvorsitzende, der sehr, sehr großen Wert legt auf Feh-
lerfreiheit und kurzen und knappen Stil, geben wir uns natürlich die größte Mühe, dem
zu entsprechen, logischerweise." (Betriebswirt, leitende Funktion in einer Versicherung)

Der Befund lässt sich verschieden deuten: Kürze und Prägnanz vermindern den an sich hohen Leseaufwand in Führungspositionen; Handlungsbedarf kann schneller erkannt werden. Ein anderer Erklärungsansatz ergibt sich mit der Rolle im Textproduktionsprozess. Teilaufgaben können an Mitarbeiter delegiert werden. Der delegierte Anteil kann sich auf das Schreiben nach Diktat beschränken, aber auch das Planen und Formulieren der Inhalte einschließen. Der Vorgesetzte bleibt in den Produktionsprozess involviert, sein Schwerpunkt verlagert sich jedoch auf das Lesen, Kontrollieren, Korrigieren und die Freigabe von Texten (durch Unterschrift) - Review und Feedback setzen die Auseinandersetzung mit Zielkriterien und Texteigenschaften voraus. Ein dritter Ansatz ergibt sich mit Ermüdungserscheinungen: Wer sich häufig durch mangelhafte Texte quälen muss, wird sensibel für Textqualitäten.

In bestimmten Kontexten geht es primär um Kürze und Prägnanz, in anderen eher um Qualitäten wie Nachvollziehbarkeit oder Vollständigkeit der Sachverhaltsdarstellung. Die Orientierung auf den Adressaten variiert berufs- bzw. domänenspezifisch. Ein wichtiger Aspekt ist die subjektive Bewertung der Schreibaufgabe.

\title{
2.3 Die Einstellung: Schreiben als Nebenprodukt
}

In einigen Berufen ist das Verfassen von Texten eine zentrale Arbeitsaufgabe (Gruppe 1). Ein Patentanwalt beschreibt z.B. seine Tätigkeit mit den Worten „Wir verkaufen beschriebenes Papier.“ In anderen Arbeitskontexten 
wird es als „uneigentliche“, als der „eigentlichen“ Arbeit nachgeordnete Aufgabe gesehen, die erledigt werden muss, aber von der eigentlichen Arbeit abhält und daher eher ungern absolviert wird, so z.B. häufig von Ärzten (Gruppe 2).

Der subjektiv wahrgenommene Stellenwert der Schreibaufgabe wirkt sich auf den Produktionsprozess aus (vgl. Couture/Rymer 1993). Während Vertreter der ersten Gruppe alle Phasen des Produktionsprozesses ähnlich sorgfältig realisieren, investieren Vertreter der zweiten Gruppe deutlich weniger Zeit und Energie in die Überarbeitung. Bei Routineaufgaben wird der Text nur selten kontrolliert und überarbeitet, etwa unter dem Aspekt seiner Verständlichkeit. Bei jedem zweiten Berufsausübenden dieser Gruppe ist der erste Entwurf zugleich der letzte. Dies gilt laut Couture/Rymer (1993) unabhängig von dem konkreten Beruf und der zu produzierenden Textsorte.

Die Konzentration auf die „eigentliche“ Arbeit (eine Diagnose stellen, Vorgänge amtlich regeln, eine Technologie entwickeln) bestimmt häufig die Perspektive auf den zu kommunizierenden Gegenstand. Dies kann trotz intendierter Verständlichkeit zu Un- oder Schwerverständlichkeit des Textprodukts führen. Ich möchte dies am Beispiel von Ingenieuren erläutern.

Ingenieure sind systemorientiert. Ihre Aufgabe besteht darin, für technische Probleme praxistaugliche Lösungen zu entwickeln. Im Vordergrund steht die Lösung, nicht der Anwender. Diese Position bestimmt die Wahl der Perspektive, z. B. bei der Dokumentation technischer Produkte. Sie wird geleitet von der Maxime der sachlichen Richtigkeit der Darstellung und der Überzeugung: „Wenn die Darstellung die Lösung korrekt wiedergibt, muss auch der Text in Ordnung sein. "In dieser Konzeptualisierung fehlt der Adressat oder er wird zweckrational gesetzt nach dem Prinzip: „Wer den Text nicht versteht, versteht nichts von der Sache. Wer nichts von der Sache versteht, ist nicht mein Adressat.".

Fehlende Textverständlichkeit ergibt sich hier nicht aus dem Unwillen, verständlich zu schreiben, sondern aus der gewählten Perspektive und der daraus resultierenden Objektfokussierung. Sie erklärt u.a., warum Anwender Produktdokumentationen, die von Ingenieuren verfasst wurden (ca. $80 \%$ aller Dokumentationen), häufig als mangelhaft oder unbrauchbar bewerten. Anwender interessiert nicht das System an sich, sondern dessen Eignung für die Erfüllung von Nutzerzielen und -zwecken (Jakobs 2000).

\subsection{Schwierige Themen, konfligierende Ziele}

Folgt man der These, dass Texte als kommunikative Artefakte an Typen von Situationen gebunden sind und die Frage nach ihrer Qualität sich daran bemisst, wie sich das Artefakt in der Situation verhält, in der es zum Einsatz kommt, dann zeigt sich relativ schnell, dass es im Beruf vielfach nicht primär um Textverständlichkeit geht. Texte dienen häufig (auch) der Absicherung der Person des Schreibers, sie sind Mittel der Klärung von Sachfragen und 
Arbeitsabläufen (Jakobs 2005a) sowie Instrumente der Beziehungsgestaltung.

Nur wenige der Befragten nennen Verständlichkeit als Anforderung oder Problem. Thematisiert werden dagegen Formulierungsprobleme bei schwierigen Themen und konfligierenden Zielen (konventionell textsortenspezifisch vereinbarte Ziele vs. Ziele des Schreibers).

$\mathrm{Zu}$ den schwierigen Themen gehört die Kommunikation unangenehmer oder ethisch sensibler Sachverhalte, wie das Mitteilen von Befunden in der medizinischen Kommunikation. Hier konfligiert häufig das Kriterium der Verständlichkeit im Sinne von Eindeutigkeit und Prägnanz mit dem der Beziehungsgestaltung durch Empathie, das Andeuten schwieriger Sachverhalte oder das Verpacken schlechter Nachrichten für Menschen, die unter emotionalem Druck stehen.

Vor allem Mehrfachadressierung erzeugt Formulierungsprobleme, so z. B. bei Laborberichten zu humangenetischen Befunden, die der behandelnde Arzt und der Patient lesen. Sie werden standardisiert verfasst, was Teilprozesse wie Informationsauswahl und -strukturierung erleichtert, verlangen jedoch bezogen auf die Zielgruppen spezifische Formulierungsleistungen.

Das erste Problem ergibt sich mit der Zielgruppe behandelnder Arzt, die einerseits möglichst kurz und knapp die wichtigsten Fakten und Interpretationen wissen will, sich jedoch abhängig vom fachlichen Hintergrund (und Jahrgang) mit geringem humangenetischem Spezialwissen ausgestattet mit komplexen, interpretationsbedürftigen Befunden auseinandersetzen muss:

„[...] die gern so einzeilige Ergebnisse hätten. Dem werd ich natürlich [...] nicht gerecht, weil die Analyse [...] einer Einzeiligkeit nicht entspricht. Es gibt häufig keine Schwarzweiß-Ergebnisse, und da muss man die schon damit belasten, dass es da einen Interpretierungsrahmen gibt. Und das mute ich den Lesern schon zu. Wobei das natürlich für alle Beteiligten anstrengender ist, aber die Vereinfachung oder die Reduzierung auf ein, zwei Sätze würde im Prinzip auch einen Fehler darstellen." (Biologin - Laborleiterin Humangenetik)

„Und wenn's dann noch [...] der Allgemeinmediziner liest und der jetzt ... Im Studium kriegen wir nicht unbedingt so viel mit von der Genetik. Das wird jetzt zum Glück [...] besser. Aber sagen wir, wenn die vor zwanzig, dreißig Jahren [...], dann wissen die so grad mal was 'n Chromosom ist. Also mehr wissen die auch nicht." (Arzt - Klinik für Humangenetik)

Die zweite Klippe ergibt sich mit der Zielgruppe Patient, die diese Berichte durchaus sieht, mit ihrem Wissen und ihrer individuellen Betroffenheit, etwa bei der genetischen Beratung von Eltern mit Kinderwunsch. Die aus der Sicht der Zielgruppe behandelnder Arzt gewünschte Kürze und Direktheit des Ausdrucks ist im Falle der Zielgruppe Patient häufig unangemessen:

„Schwerer sind die [Formulierungen], wenn ich [...] weiß, der Befund könnte an die Eltern gehen. Wo man pathologische Gegebenheiten schildern muss, ohne stigmatisierend zu sein. Stigmatisierung von Menschen zu vermeiden, das find ich sehr schwierig. Also dieses Feingefühl zu behalten. Ich meine, man untersucht Menschen; und dass 
da noch so ein bisschen rauskommt, dass es um Menschen geht und nicht um einen Sachverhalt, diese Gratwanderung, die ist schon ..." (Biologin - Laborleiterin Humangenetik)

„Es ist viel leichter, einem Kollegen einen Brief zu schreiben [...]. Du kannst natürlich alles erläutern, aber im Gespräch ist es sehr, sehr viel einfacher möglich, dass du dann einfach umschreibst. Aber das ist nicht immer so einfach, dass du sagst, arterielle Hypertonie ist Bluthochdruck. Das ist einfach, aber du musst den Leuten das natürlich so versuchen mit auf den Weg zu geben, dass die dann das Maximale daraus rausziehen und dass die es verstehen. Und das ist dann dadurch, dass das meistens nicht ganz einfache Zusammenhänge sind, [...] recht komplex. Und ich glaube, da geben sich manche Leute auch Illusionen hin, dass das gelingt. Also, dass es häufig gelingt. Es gelingt im Gespräch wesentlich einfacher, in dem du vor denen sitzt und denen [...] noch was aufzeichnest und danach haben sie's verstanden. Den Brief müsste dann eventuell der betreuende Arzt zusammen mit denen durchgehen. Manchmal haben wir dann aber auch Bedenken, dass selbst der's nicht versteht. Und die Patienten haben ja nicht alle eine gute Ausbildung. Eventuell sind's jetzt schlecht sprechende Ausländer. Und dann ist [...] schon das Beratungsgespräch schwierig, und wenn du dann mit denen über Sachverhalte sprichst, die denen eh schon schwer verständlich sind, jetzt durch die Sprache und dann auch noch vom Intellekt. Das wird dann doppelt schwer, wenn die einen deutschen Brief sehen.“ (Arzt - humangenetisches Labor)

Die Aufgabe, etwas diplomatisch oder - wie es im Zitat heißt - mit Feingefühl auszudrücken, wird in verschiedenen Zusammenhängen als schwer bezeichnet. Taktvolles Formulieren gehört meines Wissens zu den Stilvarianten, die eher wenig untersucht sind und im Gegensatz zu den Zeiten des klassischen Bildungsbürgertums auch selten gelehrt werden.

Mitunter ist es auch die Praxis, genauer: die Diskrepanz zwischen dem idealtypischen Konstrukt beruflicher Kommunikation und der Kommunikationspraxis, die die Orientierung auf den vereinbarten Adressaten und konventionelle Ziele, wie z. B. Verständlichkeit, in den Hintergrund rücken lassen. Dies zeigt sich deutlich in Interviews mit Berufsgruppen, die in Risikosituationen arbeiten, z. B. Rettungssanitäter, Feuerwehrleute und Krankenschwestern.

Rettungssanitäter dokumentieren den Einsatz noch während der Fahrt handschriftlich im Wagen. Der mit dem Patient als Teil des Einsatzformulars zu übergebende Bericht ist mehrfachadressiert: Er informiert das Krankenhauspersonal über den Zustand des Patienten am Einsatzort sowie die eingeleiteten Maßnahmen, dokumentiert den Vorfall für die Patientenakte, dient der Versicherung als Leistungsnachweis und sichert den Rettungssanitäter rechtlich ab. Aus der Sicht des Krankenhauspersonals, das unter Zeitdruck arbeitet, soll der Bericht kurz und prägnant sein, aus der Sicht der Eigenabsicherung kann er nicht ausführlich genug sein. Krankenkassen werden zwar als Adressat wahrgenommen, jedoch nicht unter dem Aspekt der Textverständlichkeit - sie scheinen die Berichte kaum zu lesen:

„Im Endeffekt bekommen die Krankenkassen die Schreiben. Die Protokolle dienen besonders auch als Selbstschutz für uns, weil wir bei Fehlern natürlich haftbar gemacht 
werden können. Deshalb müssen die Protokolle so ausführlich wie möglich sein. [...] Was die Krankenkassen damit machen, weiß ich nicht. Jedenfalls scheinen die dafür Zuständigen die Protokolle nicht gerade gründlich zu lesen. Da kamen schon Nachfragen nach dem Zustand von Patienten, obwohl im Protokoll ausdrücklich stand, dass der Patient noch auf dem Weg zum Krankenhaus verstorben ist." (Rettungssanitäter)

Der Aspekt der Absicherung durch Dokumentation wird in verschiedenen beruflichen Kontexten auffällig oft geäußert:

„Vor allem geben diese Formulare Rückendeckung. Durch 'nen Vordruck kann man sagen, wo ist es passiert, was und wie. Sonst war es Auge in Auge. Immer Aussage gegen Aussage. Und so kann man jetzt sagen, guck, so war es! Und man bekommt Recht, Rückendeckung. Das gilt für alle Formulare, die wir haben." (Feuerwehrmann)

„Die meisten Sachen gehen einfach nur in die Akten rein, also das ist einfach nur eine Absicherung [...]. Wenn wir irgendwann mal verklagt werden, gibt es ja auch immer mal wieder, dann werden diese Dinge rausgezogen und dann geht's da um die Wurst. Also ob man da irgendwas falsch dokumentiert hat, oder nicht dokumentiert hat, da kann es einem dann an den Kragen gehen." (Krankenschwester - Intensivstation)

Juristische Personen werden zu einer wichtigen Zielgruppe, z. T. zu Primäradressaten. Dies erzeugt ein Formulierungsproblem: Der Betroffene will sich juristisch „klar" ausdrücken, weiß aber oft nicht, was das im Einzelnen heißt und wie es zu realisieren ist:

„Leicht fällt mir die Krankenbeobachtung, schwer fallen mir teilweise die Pflegeberichte, wenn es dann ins juristische Detail gehen muss." (Krankenschwester - Stationspflege)

Das Problem der Mehrfachadressierung ist damit nicht zuletzt ein stilistisches Problem (stilistische Konsistenz). Dokumentationen sollen konventionell vereinbart sachlich-neutral formuliert werden. Ungeachtet dessen wird abteilungsintern mitunter ein eher informeller Stil präferiert. Er erleichtert den Betroffenen das Formulieren, konfligiert jedoch mit dem Anspruch eines „juristisch klaren“, korrekten Stils. Stilkonflikte ergeben sich nach Aussagen der Befragten vor allem im Falle innerer Betroffenheit:

\footnotetext{
„Wo ich manchmal Probleme hab, [...] ist, wenn man etwas dokumentieren muss, was etwas unangenehm ist, weil es sich um einen unangenehmen Patienten handelt oder weil Angehörige unangenehm auftreten uns gegenüber. Die zu kritisch sind uns gegenüber, die vielleicht irgendwas zu meckern haben immer, aber man will es irgendwie so dokumentieren, dass da so eine bestimmte Kritik vorhanden ist, aber man versucht sich halt auch [...] zu rechtfertigen, also wenn es gravierende Dinge sind, muss man das auch ordentlich aufschreiben. Da ist natürlich immer diese Formulierungsweise schwierig, weil, da denkt man sich dann auch, du kannst das jetzt nicht so umgangssprachlich niederschreiben, weil, es könnte immerhin sein, dass irgendjemand beim Gericht das irgendwie in der Hand hat. Wenn du dann schreibst ,der guckt doof", [...] das ist dann schon wieder schwierig, das diplomatisch zu formulieren." (Kinderkrankenschwester Intensivstation)
} 


\subsection{Arbeitsbedingungen: Stress, Zeitdruck, psychische Belastung}

Wie Schreibprozesse und deren Produkt ausfallen, hängt häufig von situativen Parametern ab. Die meisten Schreiber brauchen Zeit und Ruhe, um Texte in der gewünschten Qualität verfassen zu können, die wenigsten haben sie. Geschrieben wird in allen möglichen und unmöglichen Situationen, bei der Aufsicht im Klassenzimmer, auf dem Weg zur Arbeit im Zug, auf den Knien im Einsatzwagen. Die Kombination von Stressfaktoren wie Zeitdruck und physische und/oder psychische Belastung erschweren das Sammeln, Strukturieren und Formulieren von Informationen.

„,...] unter einer gewissen Stresssituation [...] die passenden Worte zu finden, die treffend sind, das ist immer das Problem. [...] [Im Büro] hat man Vorgaben, aber man hat Ruhe. Man kann viel denken, man kann lange denken, man kann überlegen, überlegen, überlegen, man kann sich Informationen ran holen. Nur im Rettungsdienst ist das nicht möglich. Da muss man von jetzt auf gleich die Sache fertig haben." (Feuerwehrmann - Rettungsteam)

Zeitlicher, räumlicher und emotionaler Stress werden vor allem dann zu Störfaktoren, wenn es um Texte mit weitreichenden Folgen geht, so etwa in der Kriminalistik.

„Das ist natürlich ein Unterschied, ob man tagsüber schreibt, oder ob man nachts um drei Uhr rausgeklingelt wird und kriegt dann irgendeinen Sachverhalt an den Kopf geworfen und muss dazu dann was schreiben, dann ist man ja wesentlich unkonzentrierter und ist auch davon ein bisschen abhängig, was auf einen einströmt, wer vor einem sitzt und wie man selber die Sache angeht. Wenn man vorher eine belastende Sache bearbeitet hat, hat da mit einem Toten rumgefuhrwerkt oder [...] ein Gewaltverbrechen, wo jemand verletzt worden ist, ist man natürlich nicht so frei in seinen Formulierungen, als wenn man da was morgens um halb acht noch schreibt, weil man es halt noch fertig schreiben muss." (Mitarbeiter - Kriminalpolizei)

\subsection{Domänenspezifische Tendenzen: Industrialisierung des Schreibens}

Abschließend sei ein gänzlich anderer Faktor der Arbeitsorganisation erwähnt, der (domänenspezifisch) den Aspekt der Textverständlichkeit betrifft: Die Tendenz zur Industrialisierung des Schreibens.

Die berufliche Kommunikation unterliegt wie andere Arbeitsaufgaben dem Gebot der Effizienz. Die zu erbringende Leistung soll möglichst kosten- und ressourcensparend erbracht werden. Viele Textproduktionsprozesse werden deshalb standardisiert und/oder automatisiert. Eine wesentliche Voraussetzung ist die Modularisierung der Textprodukte, die die Wiederverwendung von Textteilen und/oder ihre maschinelle Verknüpfung ermöglicht (häufig in Verbindung mit standardisierten Textstrukturen). Nickl (2005) interpretiert die genannten Phänomene - Standardisierung, Modularisierung und Automatisierung - als Ausdruck der zunehmenden Industrialisierung beruflichen 
Schreibens. Sie zeigen sich besonders deutlich im Bereich informierender Texte. $^{3}$

Die Industrialisierung des Schreibens hat Vorteile, aber auch Kosten, etwa im Falle der Nutzung von Textbausteinen. Vorteile ergeben sich aus der Minimierung individueller Formulierungsleistungen. Textbausteine reduzieren - in Kombination mit standardisierten Textstrukturen - den intellektuellen Aufwand, z. B. bei der Planung von Formulierungen, ein Aspekt, den viele Berufsausübende schätzen. Der einmal formulierte Sachverhalt wird als Textbaustein abgespeichert und bei Bedarf abgerufen, z. B. beim Schreiben von Gutachten in ärztlichen Praxen und Ingenieurbüros, beim Aufsetzen von Verträgen in Kanzleien, bei amtlichen Bescheiden in Behörden oder bei Kundenbriefen in Versicherungen und Banken.

Ein anderes Motiv für die Nutzung von Textbausteinen ist das der Mehrfachverwertung. In der technischen Dokumentation können als Baustein formulierte Warnhinweise für verschiedene Textsorten (z. B. Bedienungsanleitung vs. Sicherheitshandbuch) genutzt werden. Der Arbeitsaufwand beschränkt sich auf die (automatische) Auswahl, Kombination und Verknüpfung von Textbausteinen sowie die Zuordnung von Layoutanweisungen.

Textbausteine haben aber auch - und hier kommt Textverständlichkeit ins Spiel - eine Reihe von Nachteilen:

- Standardisierte Texte und Textbausteine schränken den Formulierungsraum des Schreibers stark ein. Die Wiederverwendung des Vorhandenen erzeugt Ähnlichkeit der Textprodukte, genauer: Merkmale wie Monotonie und Uniformität, die domänenspezifisch unterschiedlich zu bewerten sind: In der Technische Dokumentation wie auch in rechtlich geregelten Verwendungskontexten ermöglicht das Verfahren, Fehler durch das Minimieren individueller Eingriffe zu vermeiden. Im Dienstleistungsbereich sind Monotonie und Uniformität dagegen Textmerkmale, die sich eher schlecht mit dem Gedanken der Kundenorientierung vertragen. Monotonie vermindert die Lesebereitschaft wie auch die Konzentration beim Lesen, was sich auf die Verstehensleistung auswirken kann.

- Textbausteinbestände entwickeln schnell ein Eigenleben. Sie bedürfen der ständigen Pflege und Aktualisierung. Dazu fehlen im Berufsalltag die Zeit und die Ressourcen. In der Folge kommt es zu veralteten Textbausteinbeständen und Wildwuchs, so z. B. vielfach in Verwaltungen (vgl. Grönert 2004), Versicherungen und Banken. Veraltete Textbausteine erzeugen häufig inhaltliche Verständnisprobleme (der beschriebene Sachverhalt existiert nicht mehr; das Beschriebene kann vom Leser nicht nachvollzogen werden). Sie tragen zudem als Formulierungskonserven zum Überleben des Beamtendeutsch bei. Nach Grönert (2004) monieren etliche der 3 Bezogen auf Call-Center sind in der mündlichen Berufskommunikation ähnliche Ent-
wicklungen erkennbar (vgl. Nickl 2005). 
von ihr befragten Verwaltungsmitarbeiter den Stil der Textbausteine, mit denen sie arbeiten; sie sind jedoch nicht bereit, autorisiert oder fähig, diese zu verändern.

- Textbausteine eignen sich für gleiche, wiederholt auftretende Sachverhalte und Situationen. Viele kommunikative Situationen ähneln sich jedoch nur bedingt. Der Schreiber muss den Baustein der Situation, dem Gegenstand oder dem Adressaten angemessen verändern. Individuelle Veränderungen sind zeitraubend und/oder verlangen ungewohnte Formulierungsleistungen. Die geänderte Passage soll sich möglichst nahtlos in die vorgefertigte Umgebung einfügen. Dies setzt voraus, dass ein bestimmter Stil erkennbar ist. Der Sachbearbeiter muss überdies in der Lage sein, diesen Stil anzuwenden bzw. zu kopieren. Viele Berufsausübende sind auf diese Anforderungen nicht vorbereitet. Das Ergebnis sind stilistische, grammatische, argumentative Textbrüche, z.B. in den Kundenbriefen von Banken, die zu Verständnisproblemen sowie Beschwerden und - über den damit verbundenen Aufwand - zu finanziellen Verlusten führen.

Die Mehrfachverwendung durch Kombinatorik setzt voraus, dass die zu kombinierenden Bausteine inhaltlich und sprachlich autonom formuliert sind (z.B. frei von Bausteingrenzen überschreitenden anaphorischen und kataphorischen Bezügen). Der Baustein muss für Zwecke der Mehrfachverwendung (verschiedene Medien, Textsorten, Adressaten) möglichst kontext-, medien- und adressatenunabhängig formuliert werden. Versteht man Textverständlichkeit als relationales Phänomen, d.h. als Eigenschaft, die Rezipienten einem Text abhängig von ihren Voraussetzungen, Wünschen und Zielen, der vorliegenden Textsorte und dem genutzten Medium zuschreiben, ist Textverständlichkeit im oben beschriebenen Fall ein schwer zu realisierendes Konstrukt - ihm fehlen die dafür notwendigen funktional-kommunikativen Bezugs- und Bestimmungspunkte.

\section{Fazit und Ausblick}

Zusammenfassend ergibt sich im Falle des Schreibens im Berufsalltag ein breit gefächertes Bild z.T. sehr unterschiedlicher oder gar gegenläufiger Phänomene. Schreibprozesse vollziehen sich in einem komplexen Bedingungsgefüge, das Textverständlichkeit häufig erschwert und/oder in den Hintergrund treten lässt. Viele dieser Rahmenbedingungen, wie z. B. Textsorten- und Domänenspezifik, Kommunikationsaufgabe und Mehrfachadressierung, werden in psychologischen Modellen der Textverständlichkeit nach wie vor nicht berücksichtigt. Eher linguistische Bestimmungsansätze (etwa Biere 1996) gehen differenzierter vor, werden jedoch außerhalb der Linguistik - z. B. in der beruflichen Aus- und Weiterbildung - eher wenig wahrgenommen. Teilaspekte wie das Berücksichtigen adressatenspezifisch variierender Anforderungen an Textverständlichkeit bei Mehrfachadressierung oder das Bestimmen von 
Bezugspunkten und Strategien für Textverständlichkeit bei stark automatisierter Textproduktion bedürfen generell der weiteren Forschung.

Untersuchungen zeigen zudem, dass die Fähigkeit zu kommunikativem Handeln im Beruf erst im Verlauf des Berufslebens erworben wird (MacKinnon 1993, Scheller 2005). Wichtige Voraussetzungen sind das Bewusstwerden und Verstehen von Kommunikationsstrukturen, Argumentationsweisen und Aufgabenverteilung in der Organisation, die McKinnon (1993, S. 51) als „contextualizing knowledge " bezeichnet. $\mathrm{Zu}$ diesem Wissen gehört das Begreifen des Arbeitsplatzes als Diskursgemeinschaft, das Bewusstsein, dass der Leser ein bestimmtes kommunikatives Bedürfnis hat, Wissen über die Funktionen der eigenen Texte im übergeordneten Zusammenhang der Organisation, Erfahrungen im Feedback-Verarbeiten und -Geben und vieles andere mehr.

Der Prozess der allmählichen Entwicklung beruflicher Formulierungsexpertise sollte systematisch durch Ausbildungsangebote unterstützt und begleitet werden. Hier zeichnet sich insgesamt ein erheblicher Handlungsbedarf $a b$, so z.B. in der Entwicklung von Vermittlungs- und Trainingskonzepten für adressaten-, aufgaben- und situationsangemessenes sprachliches Handeln. Dazu gehören u. a. auch das Formulieren schwieriger Themen, „diplomatisches“ Formulieren oder taktvolles Formulieren. Die Linguistik kann diese Defizite ausgleichen und damit einen wichtigen Beitrag für die Wirtschaft leisten.

\section{Literatur}

Aebli, Hans (1983): Zwölf Grundformen des Lehrens. Stuttgart: Klett Cotta.

Biere, Bernd Ulrich (1996): Textgestaltung zwischen Sachangemessenheit und Adressatenorientierung. In: Krings, Hans P. (Hg.): Wissenschaftliche Grundlagen der Technischen Kommunikation. Tübingen: Narr. S. 291-306.

Couture, Barbara/Rymer, Joan (1993): Composing processes on the job by writer's role and task value. In: Spilka, Rachel (ed.): Writing in the workplace. Carbondale, Edwardsville: Southern Illinois University Press. S. 420-433.

Fix, Ulla (2005): Was heißt Texte kulturell verstehen? Ein- und Zuordnungsprozesse beim Verstehen von Texten als kulturellen Entitäten. In diesem Band.

Grönert, Kerstin (2004): Verständigung und Akzeptanz in der Kommunikation zwischen Bürger und Verwaltung. Beobachtung und Analyse der kommunikativen Interaktion anhand von Bescheiden und Formularen. Diss., Universität Bielefeld, http//bieson.ub. uni-bielefeld.de/volltexte/2004/534.

Jakobs, Eva-Maria (1999): Textvernetzung in den Wissenschaften. Zitat und Verweis als Ergebnis rezeptiver, reproduktiver und produktiver Prozesse. Tübingen: Niemeyer.

Jakobs, Eva-Maria (2000): Technische Dokumentation. In: Becker-Mrotzek, Michael/Brünner, Gisela/Cölfen, Hermann (Hg.): Linguistische Berufe. Ein Ratgeber zu aktuellen linguistischen Berufsfeldern. Frankfurt/M. u.a.: Lang. S. 71-78.

Jakobs, Eva-Maria (2005a): Writing at Work. In: Jakobs, Eva-Maria/Lehnen, Katrin/ Schindler, Kirsten (Hg.): Schreiben am Arbeitsplatz. Wiesbaden: US Verlag für Sozialwissenschaften. S. 13-40.

Jakobs, Eva-Maria (2005b): Telefon, Email oder Fax? Medial gestützte Kommunikation in Unternehmen. In: Perrin, Daniel/Kessler, Ursula (Hg.): Schreiben im Netz. Wiesbaden: US Verlag für Sozialwissenschaften. S. 26-40. 
Keller, Rudi (2004): Die Sprache des Geschäftsberichtes. www.phil-fak.uni-duesseldorf.de/ rudi.keller/index.php?spragebe.php\&1.

Langer, Inghard/Schulz von Thun, Friedemann/Tausch, Reinhard (1975): Sich verständlich ausdrücken. Basel/München: Ernst Reinhardt.

MacKinnon, Jamie (1993): Becoming a rhetor: Developing writing ability in a mature, writing-intensive organization. In: Spilka, Rachel (ed.): Writing in the Workplace. New Research Perspectives. Carbondale/Edwardsville: Southern Illinois University Press. S. 41-55.

Nickl, Markus (2005): Industrialisierung des Schreibens. In: Jakobs, Eva-Maria/Lehnen, Katrin/Schindler, Kirsten (Hg.): Schreiben am Arbeitsplatz. Wiesbaden: US Verlag für Sozialwissenschaften. S. 43-56.

Scheller, Petra (2005): Schreibkompetenz in ingenieurwissenschaftlichen Berufen. Magisterarbeit, Fakultät 7 der RWTH Aachen, Institut für Sprach- und Kommunikationswissenschaft, Abt. Technik-Kommunikation.

Wagner, Jörg (2002): Mensch-Computer-Interaktion. Sprachwissenschaftliche Aspekte. Frankfurt/Main: Lang. 